\title{
Long-term monounsaturated fatty acid diets reduce platelet aggregation in healthy young subjects
}

\author{
Ruth D. Smith ${ }^{1}$, Colette N. M. Kelly ${ }^{1}$, Barbara A. Fielding ${ }^{2}$, David Hauton ${ }^{2}$, \\ K. D. Renuka R. Silva ${ }^{1}$, Margaretha C. Nydahl ${ }^{1}$, George J. Miller ${ }^{3}$ and Christine M. Williams ${ }^{1 *}$ \\ ${ }^{1}$ Hugh Sinclair Unit of Human Nutrition, School of Food Biosciences, University of Reading, Reading RG6 6AP, UK \\ ${ }^{2}$ Oxford Lipid Metabolism Group, Nuffield Department of Clinical Medicine, University of Oxford, Oxford OX2 6HE, \\ UK
}

${ }^{3}$ Epidemiology and Medical Care Unit, Medical Research Council, Wolfson Institute of Preventative Medicine, Charter House Square, London EC1M 6BQ, UK

(Received 18 July 2002 - Revised 20 May 2003 - Accepted 2 June 2003)

\begin{abstract}
The aim of the present study was to compare the response of a range of atherogenic and thrombogenic risk markers to two dietary levels of saturated fatty acid (SFA) substitution with monounsaturated fatty acids (MUFA) in students living in a university hall of residence. Although the benefits of such diets have been reported for plasma lipoproteins in high-risk groups, more needs to be known about effects of more modest SFA-MUFA substitutions over the long term and in young healthy adults. In a parallel design over 16 weeks, fifty-one healthy young subjects were randomised to one of two diets: (1) a moderate-MUFA diet in which $16 \mathrm{~g}$ dietary SFA/100 g total fatty acids were substituted with MUFA ( $n$ 25); (2) a high-MUFA diet in which $33 \mathrm{~g}$ dietary SFA/100 g total fatty acids were substituted with MUFA ( $n$ 26). All subjects followed an 8-week run-in diet (reference diet), with a fatty acid composition close to the UK average values. There were no differences in plasma lipid responses between the two diets over 16 weeks of the study with similar reductions in total cholesterol $(P<0.001)$ and LDL-cholesterol $(P<0.01)$ in both groups; a small but significant reduction in HDL-cholesterol was also observed in both groups $(P<0.01)$. Platelet responses to ADP $(P<0 \cdot 01)$ and arachidonic acid $(P<0.05)$ differed with time on the two diets; at 16 weeks, platelet aggregatory response to ADP was significantly lower on the high-MUFA than the moderate-MUFA $(P<0 \cdot 01)$ diet; ADP responses were also significantly lower within this group at $8(P<0 \cdot 05)$ and $16(P<0 \cdot 01)$ weeks compared with baseline. There were no differences in fasting factor VII activity (factors VIIc and VIIag), fibrinogen concentration or tissue-type plasminogen activator activity between the diets. There were no differences in postprandial factor VIIc responses to a standard meal (area under the curve) between the diets after 16 weeks, but postprandial factor VIIc response was lower than on the high-MUFA diet compared with baseline $(P<0 \cdot 01)$. In conclusion, a high-MUFA diet sustains potentially beneficial effects on platelet aggregation and postprandial activation of factor VII. Moderate or high substitution of MUFA for SFA achieves similar reductions in fasting blood lipids in young healthy subjects.
\end{abstract}

Monunsaturated fatty acids: Platelet aggregation: Coagulation

Population recommendations aimed at reducing the incidence of CHD have previously emphasised the need for reductions in saturated fatty acid (SFA) intakes (National Cholesterol Education Program, 1991; Department of Health, 1994). However, progress towards achieving these population targets through reductions in total fat has been slow (Ministry of Agriculture, Fisheries and Food, 1996) and may partly reflect consumer resistance to low-fat products. In addition, there has been concern regarding potentially adverse effects of low-fat, high-carbohydrate diets, which have been shown to increase plasma triacylglycerol (TAG) and decrease beneficial HDL-cholesterol (Mensink \& Katan, 1992; Katan, 1997).
Alternative dietary strategies for reducing SFA intakes, such as the substitution of a proportion of dietary SFA with monounsaturated fatty acids (MUFA), have also been proposed. When compared with low-fat, high-carbohydrate diets, high-MUFA diets reduce total cholesterol and LDL-cholesterol without reducing HDL-cholesterol or increasing fasting plasma TAG (Grundy, 1986; Mensink et al. 1989; Kris-Etherton \& Yu, 1997). Other potential benefits of high-MUFA diets include favourable influences on haemostasis (Sirtori et al. 1986; Lopez-Segura et al. 1996), reduced susceptibility of LDL to oxidative modification (Reaven et al. 1991; Aviram \& Eias, 1993), reduced inflammatory response (Yaqoob et al. 1998) and attenuated postprandial lipaemia and factor VII responses to acute fat

\footnotetext{
Abbreviations: AUC, area under the curve; MUFA, monounsaturated fatty acid; PUFA, polyunsaturated fatty acid; SFA, saturated fatty acid; TAG, triacylglycerol; tPA, tissue-type plasminogen activator.

* Corresponding author: Professor Christine M. Williams, fax +44 118931 0080, email c.m.williams@reading.ac.uk
} 
ingestion (Roche et al. 1998; Zampelas et al. 1998; Larsen et al. 1999). The attenuated postprandial response of factor VII on a high-background-MUFA diet is of particular interest, since acute meal studies, with subjects consuming their normal background diet, have shown exaggerated postprandial factor VII activation following consumption of olive oil (Oakley et al. 1998; Sanders et al. 1999).

High levels of MUFA substitution for SFA have been used in many of the previous experimental studies, resulting in fatty acid intakes close to those of Cretan populations in the 1960s. Although levels such as these are achievable in free-living populations under experimental conditions (Williams et al. 1999), they would require considerable changes in the supply chain of fat if they were to be implemented at population level in the UK. Many of the previous studies have been conducted in middle-aged subjects or those with elevated fasting lipoprotein levels and it is not known whether the beneficial effects observed apply across all population groups.

The purpose of the present study was to compare the effects of substituting dietary SFA at two levels of dietary MUFA (moderate and high) on a range of atherogenic and thrombogenic risk markers in young adults living in a residential setting (a university hall of residence). A long-term intervention (16 weeks) was employed to determine whether effects that have been reported in short-term studies are sustained in the long term or whether there is attenuation through adaptation. To ensure adequate control over the subjects' diets and prevent loss of long-term compliance, modifications to the diet were achieved by altering the fats used in food preparation in the kitchen of the residence, and by providing the subjects with specially manufactured spreads and snack foods. In addition to the classical atherogenic lipoprotein risk markers (total cholesterol and LDL- and HDL-cholesterol), LDL sub-types (LDL1, LDL2 and LDL3) and in vitro oxidative stability of LDL were measured. Thrombogenic risk markers included measurements of factor VII, fibrinogen and tissue-type plasminogen activator (tPA). The postprandial response of factor VII to a standard meal was monitored at baseline and after 16 weeks on the two diets. It was important to assess the effects of the MUFA diets on in vitro platelet aggregation, since although the polyphenolic components of olive oil have been shown to reduce platelet aggregation (Petroni et al. 1995), claims for beneficial effects on platelet aggregation of replacing SFA with MUFA per se are not well supported by the available literature for human subjects: this is sparse and contradictory (McDonald et al. 1989; Sirtori et al. 1992; Freese et al. 1994; Turpeinen et al. 1998). Since the aim of the present study was to investigate the effects, specifically, of changes in fatty acid intake, refined oils (rapeseed and oleic acidenriched sunflower oil) were used to provide the additional dietary MUFA.

\section{Subjects and methods}

\section{Subjects}

Fifty-one students residing in a fully catered residence in the University of Reading were recruited to participate in the overall trial; forty-eight completed the protocol. This location was chosen because of the feasibility of altering long-term fat intakes by changing the fats used in the residence kitchens. The study was approved by the University of Reading Ethics Committee and all subjects gave written, informed consent to participate. Twenty-seven of the fifty-one students also participated in detailed postprandial metabolic studies and these results have been reported elsewhere (Silva et al. 2003).

\section{Recruitment}

Potential subjects were screened and recruited according to specific inclusion criteria. Subjects recruited were 18-28 years old, with BMI $20-25 \mathrm{~kg} / \mathrm{m}^{2}$, not following a diet or taking dietary supplements or medication affecting blood lipoproteins or coagulation factors (female subjects using oral contraceptives were included, but asked to continue to take them throughout the study), no endocrine or liver disease, not undertaking regular vigorous exercise (no more than $3 \times 20 \mathrm{~min}$ aerobic sessions per week), not consuming $>8 \mathrm{~g}$ alcohol/week. All subjects were healthy and normolipidaemic, having fasting lipid measurements within the reference ranges.

\section{Study design}

The study was a controlled parallel single-blind design of two MUFA diets with a run-in period of 8 weeks on a standard reference diet. The reference diet was necessary to ensure that varying dietary habits of this student population did not contribute to a variable baseline and influence the degree of response to the MUFA-rich diets. Following evaluation of habitual diet and collection of two baseline blood samples, subjects consumed an SFA-rich reference diet for 8 weeks, immediately followed by either a moderate- or high-MUFA diet for an additional 16 weeks. A randomised crossover design was not employed because of the long-term nature of the intervention. Subjects were allocated to moderate- and high-MUFA groups using stratified randomisation with gender, fasting total cholesterol and TAG as stratification factors and there were no differences between the groups on allocation to the study. Table 1 shows the anthropometric, blood biochemical and smoking characteristics, according to gender, after randomisation to the moderate- and high-MUFA groups. There were no significant differences between the two groups in any of the variables measured. The numbers of subjects who were smokers and the use of oral contraceptives were also similar between the two groups. Fasting blood samples were taken at baseline (M0) and at 8-week intervals (M8 and M16) throughout the study. Postprandial factor VIIc and factor VIIag $8 \mathrm{~h}$ responses to a standard meal were conducted at baseline (M0) and at 16 weeks (M16) in a subgroup of subjects from each group (moderate-MUFA $n$ 14, high-MUFA $n$ 15).

\section{Dietary intervention}

The run-in diet (reference diet) was designed to provide a dietary fatty acid intake close to the mean UK profile 
Table 1. Characteristics of each group according to gender after randomisation (Mean values and standard deviations)

\begin{tabular}{|c|c|c|c|c|c|c|c|c|c|c|c|c|}
\hline & \multicolumn{6}{|c|}{ Moderate-MUFA group } & \multicolumn{6}{|c|}{ High-MUFA group } \\
\hline & \multicolumn{2}{|c|}{ Males (n 13) } & \multicolumn{2}{|c|}{$\begin{array}{c}\text { Females } \\
(n 12)\end{array}$} & \multicolumn{2}{|c|}{ All $(n 25)$} & \multicolumn{2}{|c|}{ Males (n 13) } & \multicolumn{2}{|c|}{$\begin{array}{c}\text { Females } \\
(n 13)\end{array}$} & \multicolumn{2}{|c|}{ All $(n 26)$} \\
\hline & Mean & SD & Mean & SD & Mean & SD & Mean & SD & Mean & SD & Mean & SD \\
\hline Age (years) & 21 & 3 & 20 & 1 & 20 & 3 & 20 & 2 & 20 & 2 & 20 & 2 \\
\hline Weight (kg) & $72 \cdot 4$ & $6 \cdot 1$ & $59 \cdot 1$ & $10 \cdot 7$ & $66 \cdot 0$ & $10 \cdot 8$ & $69 \cdot 3$ & $6 \cdot 4$ & $59 \cdot 2$ & $11 \cdot 0$ & $64 \cdot 2$ & $10 \cdot 2$ \\
\hline BMI $\left(\mathrm{kg} / \mathrm{m}^{2}\right)$ & 22.9 & 1.9 & $21 \cdot 8$ & $3 \cdot 4$ & $22 \cdot 4$ & $2 \cdot 7$ & $22 \cdot 3$ & $2 \cdot 4$ & $22 \cdot 6$ & $3 \cdot 1$ & $22 \cdot 4$ & $2 \cdot 7$ \\
\hline Total cholesterol (mmol/l) & 3.8 & 0.5 & 4.0 & 0.7 & 3.9 & 0.6 & 3.8 & 0.6 & $4 \cdot 1$ & 0.9 & $4 \cdot 0$ & 0.8 \\
\hline HDL-cholesterol (mmol/l) & $1 \cdot 2$ & 0.4 & 1.6 & 0.3 & $1 \cdot 4$ & 0.4 & $1 \cdot 2$ & 0.2 & $1 \cdot 6$ & 0.4 & 1.4 & 0.4 \\
\hline TAG $(\mathrm{mmol} / \mathrm{l})$ & 0.9 & 0.2 & 0.7 & 0.3 & 0.8 & 0.3 & $1 \cdot 1$ & 0.5 & 0.8 & 0.6 & 1.0 & 0.6 \\
\hline Glucose (mmol/l) & $4 \cdot 8$ & 0.5 & $4 \cdot 6$ & 0.3 & $4 \cdot 7$ & 0.4 & $5 \cdot 0$ & 0.3 & $4 \cdot 7$ & 0.4 & $4 \cdot 8$ & 0.4 \\
\hline Smoker $(n)$ & 4 & & 3 & & 7 & & 4 & & 3 & & 7 & \\
\hline Oral contraceptive use $(n)$ & - & & 5 & & 5 & & - & & 5 & & 5 & \\
\hline
\end{tabular}

MUFA, monounsaturated fatty acid; TAG, triacylglycerol.

(\% energy: SFA 16, MUFA 12, polyunsaturated fatty acids (PUFA) 6). In the moderate-MUFA diet the aim was to substitute $16 \mathrm{~g}$ dietary SFA $/ 100 \mathrm{~g}$ total fatty acids with MUFA to achieve (\% energy): SFA 13, MUFA 15, PUFA 6. In the high-MUFA diet the aim was to substitute $33 \mathrm{~g}$ dietary SFA/100 $\mathrm{g}$ total fatty acids with MUFA to achieve (\% energy): SFA 10, MUFA 18, PUFA 6. A detailed account of the dietary calculations, fats and oils and methods used to achieve the target intakes of fats and fatty acids on both the SFA (control) and MUFA diets has been described in full by Nydahl et al. (2003). An important design criterion was that the diets should differ only in the proportions of SFA and MUFA, while maintaining constant intakes of energy, PUFA and trans fatty acids. It was also necessary for the palmitic:stearic acid ratios to be kept constant between the diets, due to the possible differences in plasma lipid responses to individual fatty acids within the same fatty acid class. Dietary fat intakes were altered at baseline to provide the reference diet by changing the types of fats used in the kitchens for frying, baking and spreading. In addition, students were provided with spreading fats for use outside the residence dining room, and specially prepared snacks (biscuits, cakes and crisps) and low-fat meals were also provided to aid compliance. After 8 weeks on the run-in diet, the fats and oils used in the kitchens, and spreading fats and snack foods used by the students directly, were changed to increase the MUFA contents of the background diets. Spreading and cooking fats for both SFA and MUFA diets were specially manufactured by Unilever Best Foods (Crawley, Sussex, UK) and snack foods by United Biscuits (Maidenhead, Berks., UK). Only refined oils were used throughout, and rapeseed oil and oleic acid-enriched sunflower oil were used as the source of MUFA in the cooking and spreading fats.

\section{Dietary assessment and compliance}

Compliance to the SFA and MUFA diets was monitored once in each dietary period by a $4 \mathrm{~d}$ diet diary, in which subjects were asked to record all food and drink consumed during 4 consecutive days, including one weekend day. Diet diaries were recorded for a period of $4 \mathrm{~d}$ between weeks 4 and 8 on the reference diet and between weeks 8 and 14 on the MUFA diets, according to ease of convenience for the students in relation to their study load. Subjects were instructed to record any leftovers and describe and estimate portion sizes of foods that they ate outside the dining room, including snacks, drinks and meals eaten during weekends. The investigators weighed portion sizes of food served at the residence kitchen. Further weighed intake measurements of the portions were made in the dining hall randomly. Students were required to sign in to lunch and evening meals in the hall of residence and to record their choice of menu, which rotated on a 2-week basis. Compliance was also aided by weekly contact with the volunteers; investigators attended the hall of residence at weekly intervals to provide additional snack foods and margarine, note any deviations in the use of foods from the kitchens, as well as changes in food habits due to illness or absence from the university. An objective measure of compliance was provided by measurement of plasma phospholipid fatty acid compositions.

\section{Anthropometric and biochemical measurements}

Height, weight and blood pressure were recorded at the initial screening visit. Body weights were recorded at 2-week intervals at the time of the investigators' visit to the hall of residence. Prior to all blood collections, subjects were required to abstain from strenuous exercise and alcohol consumption for $24 \mathrm{~h}$. To standardise conditions for collection of blood for haemostatic measurements, subjects were provided with a standard low-fat evening meal $(<10 \mathrm{~g}$ total fat) the day before each blood collection. Subjects were excluded from the use of aspirin throughout the whole period of study and were supplied with alternative pain relief. Blood samples for measurement of platelet aggregation and haemostatic factors were taken on separate days from those for other outcome measures to allow adequate time for collection and processing of samples. On the postprandial study days, subjects arrived at the unit at 
08.00 hours. A cannula was inserted into a forearm vein and two fasting (baseline) blood samples were collected over a period of $10 \mathrm{~min}$. A test meal, consisting of $40 \mathrm{~g}$ Rice Krispies (Kelloggs, Manchester, UK), $200 \mathrm{~g}$ whole milk, $72 \mathrm{~g}$ white bread, $14 \mathrm{~g}$ Flora $^{\mathrm{TM}}$ margarine (Unilever Best Foods, Dublin, Republic of Ireland) and $50 \mathrm{~g}$ cheddar cheese, was given. The meal provided $45.0 \mathrm{~g}$ fat (SFA $22.0 \mathrm{~g}$, MUFA $12.0 \mathrm{~g}$, PUFA $6.5 \mathrm{~g}$ ), $93 \mathrm{~g}$ carbohydrate and $33 \mathrm{~g}$ protein. Blood samples were collected for determination of factors VIIc and VIIag at $2 \mathrm{~h}$ intervals over the next $8 \mathrm{~h}$. Water was allowed, but no further food was consumed during the blood collection period. All blood samples were collected into evacuated tubes (Becton Dickinson VACUTAINER Systems, Europe; Becton Dickinson, Leicester, UK) according to a standardised protocol and with minimal venostasis.

With the exception of blood taken for measurement of haemostasis and whole blood aggregation (for details, see later), all samples were initially stored on ice and plasma separated by centrifugation at $3000 \mathrm{rpm}$ for $15 \mathrm{~min}$. Sucrose $(12 \mu \mathrm{l}$ sucrose solution $(500 \mathrm{~g} / \mathrm{l}) / \mathrm{ml}$ plasma) was added to plasma aliquots for determination of the LDL density profile. Plasma samples for analysis of lipid and the fatty acid composition of plasma phospholipids and cholesteryl esters were stored at $-20^{\circ} \mathrm{C}$. Plasma for all other analyses was stored at $-70^{\circ} \mathrm{C}$. Samples from each subject were analysed in the same batch at the end of the study.

Fasting lipid concentrations. Plasma concentrations of TAG, total cholesterol and HDL-cholesterol were determined in plasma from blood samples collected into lithium heparin-containing Vacutainer tubes (Becton Dickinson), using enzymatic colorimetric kits on a Monarch Automatic Analyser (Instrumentation Laboratories Ltd, Warrington, Ches., UK). HDL-cholesterol concentration was determined following the precipitation of LDL and VLDL from fresh plasma using dextran sulfate; the resultant supernatant fraction was frozen at $-20^{\circ} \mathrm{C}$ prior to analysis. The intra- and inter-assay CV were $<5 \%$ for all assays. LDL-cholesterol was calculated using Friedewald's formula (Friedewald et al. 1972).

LDL isolation and oxidation. Briefly, plasma $(1.75 \mathrm{ml})$ was layered under density solution $(1.006 \mathrm{~kg}$ sucrose $/ 1)$ in Beckman polyallomer tubes $(5.1 \mathrm{ml})$ and centrifuged at $100000 \mathrm{rpm}$ in a Beckman TLX ultracentrifuge for $2.5 \mathrm{~h}$ using TLA100.4 fixed-angle centrifuge rotor (Beckman, High Wycombe, Bucks, UK). The pellet was isolated from the base of the tube, and the LDL was isolated following a second flotation step, density-adjusted and layered under density solution $(1.063 \mathrm{~kg}$ sucrose/l) for a second centrifugation at $100000 \mathrm{rpm}$ for $9 \mathrm{~h}$. The volume of the recovered LDL fraction was $1.93 \mathrm{ml}$.

Oxidation of LDL using 2,2'-azobis(2-amidinopropane) (Aldrich, Poole, Dorset, UK) was carried out essentially according to the method of Volpi \& Tarugi (1999) with all oxidations tested at $37^{\circ} \mathrm{C}$. Analysis was undertaken using a Beckman DU640 spectrophotometer fitted with a multicell-transporter and auto six sampler accessories. Calculations were based upon a Microsoft Excel spreadsheet and macro obtained from Dr J. McEneny (Queen's University, Belfast, Northern Ireland, UK) (McDowell et al. 1995).
LDL density profile. LDL particle size distribution was assessed according to the method of Griffin et al. (1990), recently adapted for use in small samples (Callow et al. 1998). Density-adjusted plasma samples $(1.2 \mathrm{ml})$ were layered below a $\mathrm{NaCl}-\mathrm{KBr}$ discontinuous density gradient and centrifuged using an SW55Ti centrifuge rotor (Beckman) for $19 \mathrm{~h}$ at $20^{\circ} \mathrm{C}$ at $55000 \mathrm{rpm}$. Gradients were pumped directly out of the centrifuge tube, using an inert carrier into a spectrophotometer flow cell and the absorbance was measured at $280 \mathrm{~nm}$. From the traces obtained, the area under the curve (AUC) for each LDL sub-fraction was calculated and the results expressed as \% total.

Haemostatic variables. Timing of blood samples is critical for platelet function tests and fibrinolytic activity, with activity lowest in early morning; strict adherence to timed appointments was applied throughout the study. Thompson's (1992) guidelines for collection of blood for measurement of coagulation and fibrinolytic activity were used throughout. Experienced phlebotomists collected samples with minimal haemostasis, tourniquets were employed with minimal compression and sampling was completed within $90 \mathrm{~s}$. Avoidance of cold and contact activation of factor VII was rigorously adhered to throughout the study. Samples were collected into siliconised sodium citrate $(0.105 \mathrm{M})$-containing Vacutainer tubes (Becton Dickinson) after that taken for lipid analysis, to ensure that a free-flowing sample was obtained. Blood for determination of t-PA activity was collected into a $2.5 \mathrm{ml}$ acid citrate-containing Vacutainer tube (Becton Dickinson). All haemostatic assays were carried out at the Wolfson Coagulation Laboratory (MRC Epidemiology and Medical Care Unit, London) on samples stored at $-70^{\circ} \mathrm{C}$.

Factor VIIc was measured by a one-stage, semi-automated bioassay using rabbit-brain thromboplastin (Diagen, Thame, Oxon., UK) and a factor VII-deficient substrate plasma prepared as described by Miller et al. (1994). t-PA activity was determined by a chromogenic assay (Coatest PAI; Chromogenix AB, Sweden). Factor VII antigen was determined by using an ELISA (Asserachrom VII:Ag, Stago Diagnostica, Parsippany, NJ, USA). Fibrinogen was measured by a thrombin-clotting method using a standard plasma (Immuno, Vienna, Austria). All coagulation assays were performed in duplicate and the results expressed as the mean value. Intra- and interassay $\mathrm{CV}$ were in the range $3-13 \%$.

Whole-blood aggregation. Whole-blood aggregation was measured in a subgroup of the subjects (moderateMUFA $n$ 14, high-MUFA $n$ 15) using the impedance technique based on previously published methods (Cardinal \& Flower, 1980). Previous validation of the technique in our hands (Kelly, 2000) provided dose-response data for each agonist employed and demonstrated the need for strict adherence to a standardised protocol for collecting blood, timing of blood sampling and time lapse between analyses and quantification procedure. Under these conditions, within- and between-assay variability did not exceed $10 \%$. Results from two subjects in each group could not be used in the analyses due to unreliability of at least one sample in the series. Blood was diluted $50 \%$ with PBS (Sigma, Poole, Dorset, UK) and held at room temperature. Samples of $1 \mathrm{ml}$ were placed into plastic cuvettes 
with a stir-bar and warmed to $37^{\circ} \mathrm{C}$. The impedance probe was placed into the warmed blood, the sample was stirred at $1000 \mathrm{rpm}$ and the baseline impedance set to zero. Collagen (final concentration: $4.0 \mu \mathrm{g} / \mathrm{ml}$ ), ADP (final concentration $10 \mu \mathrm{mol} / \mathrm{l}$,) or arachidonic acid (final concentration $1.0 \mathrm{mmol} / \mathrm{l}$ ) was added to the cuvette and the impedance increased in proportion to the amount of platelet aggregation on the platelet probe. Measurements were registered after 6 min using a two-channel whole-blood aggregometer (Chrono-Log Corp., Labmedics Ltd, Romiley, Ches., UK). For each agonist, the aggregation response was analysed in duplicate and averaged. Measurements commenced within $1 \mathrm{~h}$ of blood being drawn and finished within $3 \mathrm{~h}$. Reagents were supplied by Labmedics Ltd, except collagen, which was obtained from Bio-Stat Ltd (Stockport, Ches., UK).

Plasma biomarkers. To provide an objective measure of dietary compliance, the fatty acid composition of plasma phospholipids was measured by capillary GC, as described by Fielding et al. (1996). Specific fatty acids were identified by reference to methyl ester standards and peak areas were corrected for individual response factors. Results were expressed as composition by weight ( $\mathrm{g} / 100 \mathrm{~g}$ total fatty acids). Intra and inter-assay CV were in the range $2-11 \%$. Results for plasma phospholipid and cholesterol ester compositions for the whole study sample have been reported previously (Nydahl et al. 2003). Results for plasma phospholipids of a subgroup that undertook the platelet aggregation measurements are presented in the results section of the present paper to inform the platelet aggregation results (moderate-MUFA $n$ 14, high-MUFA $n$ 15).

\section{Data analysis}

Results are shown in the Tables as mean values and standard deviations and in the Figures as mean values with their standard errors. The postprandial data (factors VIIc and VIIag) were expressed in summary form (AUC, incremental AUC). AUC was calculated using the trapezoidal method as the area between the plasma concentration and the time axis at time $t 0$; integrated AUC was calculated from AUC by correcting to individual baseline values at t 0 (Matthews et al. 1990). Sample size was estimated based on a predicted difference in cholesterol lowering between the two diets of $10 \%$, at a level of significance of $P<0.05$, with a power of $80 \%$. All statistical analysis was performed using SPSS (version 9.0; SPSS, Chicago, IL, USA). Data were checked for normality and log transformation was performed where necessary to normalise the data. For each variable, the statistical significance of time $\times$ treatment effects were analysed using two-factor repeated-measures ANOVA. When the time $\times$ treatment interactions were significant, post hoc tests (independent $t$ tests) with Bonferonni's correction were applied; changes within groups with time were assessed by paired analysis of specific time points. A value of $P<0.05$ was accepted as statistically significant.

\section{Results}

The reported energy, macronutrient and fatty acid intakes of the two groups during both the reference and MUFA diets are shown in Table 2. SFA intake was significantly lower on the high-MUFA than the moderate-MUFA diet $(P<0 \cdot 001)$, but differences in MUFA intakes between the two MUFA groups did not quite reach statistical significance $(P<0 \cdot 06)$. Compared with the reference diet, the MUFA dietary interventions achieved significant increases in cis-MUFA intake $(P<0 \cdot 001)$ and reductions in SFA intake $(P<0.001)$, whilst keeping energy, total fat, cisPUFA and trans fatty acid intake consistent as the subjects transferred from the run-in reference diet to their respective MUFA diets. Changes achieved were close to target values, with mean decreases in SFA intakes of 21 (SD 3) and 32 (SD 5) $\%$ and increases in MUFA intakes of 23 (SD 4) and 37 (SD 6) \% in moderate- and high-MUFA groups respectively.

The fatty acid compositions of plasma phospholipids were determined to provide an objective measure of dietary

Table 2. Reported macronutrient and fatty acid intakes on reference and monounsaturated fatty acid (MUFA) dietsł

(Mean values and standard deviations)

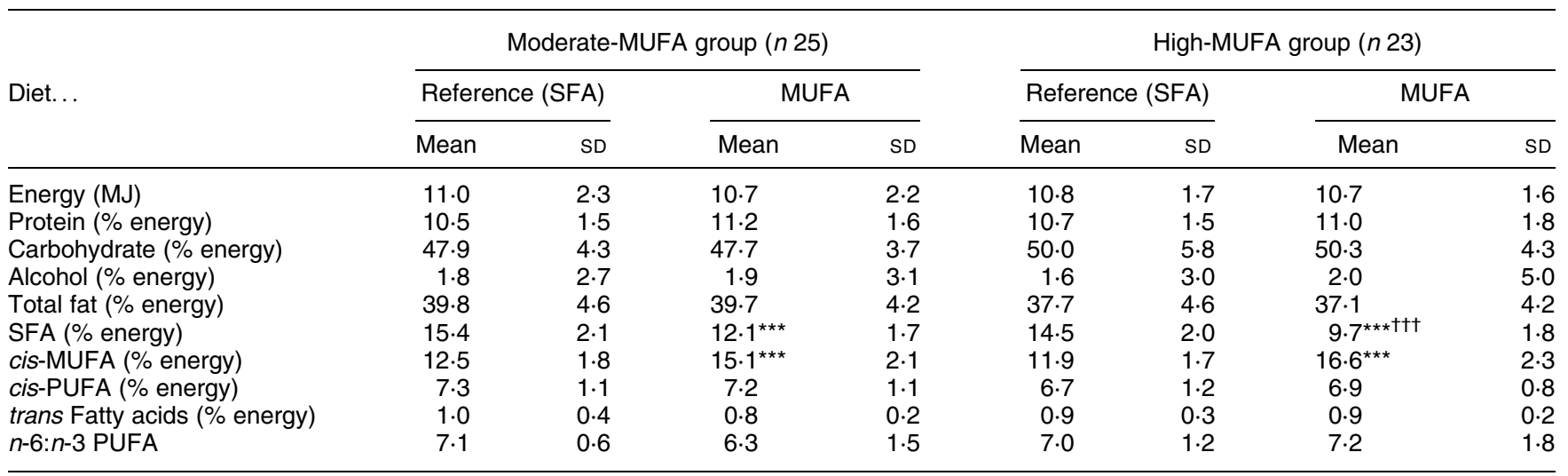

SFA, saturated fatty acid; PUFA, polyunsaturated fatty acid.

Mean values were significantly different from those of the reference diet within the same group: ${ }^{\star \star \star} P<0.001$.

Mean value was significantly different from that of the moderate-MUFA group: ${ }^{+\dagger} P<0.001$.

$\ddagger$ For details of subjects, diets and procedures, see Table 1 and p. 598. 
compliance (Table 3). This plasma biomarker showed anticipated patterns of change, with SFA content tending to decrease on the MUFA diets with the opposing trend for MUFA content. There were no significant differences in phospholipids fatty acid compositions (diet $\times$ time interactions) between the two diets. Compared with the reference diet, changes in fatty acid composition on the high-MUFA diet, but not the moderate-MUFA diet, reached significance for phospholipid total MUFA at $8(P<0.05)$ and $16(P<0.01)$ weeks. In the case of individual fatty acids, and for the high-MUFA group only, 16:0 showed a significant decline at $8(P<0.01)$ and $16(P<0.05)$ weeks, whilst $18: 1$ showed corresponding increases that were significant at 16 weeks $(P<0 \cdot 05)$. It was notable that although there were no significant changes in total PUFA phospholipids, levels of $18: 2$ were significantly reduced at 8 and 16 weeks on the high-MUFA diet $(P<0 \cdot 01)$.

Fasting plasma lipids, LDL sub-fractions and LDL oxidative stability (lag time) are shown in Table 4. Repeated-measures ANOVA showed no differences between the diets in terms of these outcome measures with time. Both MUFA dietary groups showed significant reductions in total cholesterol $(P<0.001)$ and LDL-cholesterol $(P<0.01)$ with gradual reductions observed over 8 and 16 weeks. However, in the last 8 weeks of both diets there were also small but significant reductions in HDLcholesterol $(P<0 \cdot 01)$, so that by the end of the 16 -week diets there were no significant changes in the total cholesterol:HDL-cholesterol ratios.

Repeated-measures ANOVA revealed no differences in the proportions of the major LDL sub-fractions (LDL1, LDL2, LDL3) between the diets with time, although in the moderate MUFA group, analysis of specific timepoints revealed a significant increase in LDL1 from baseline to week $16(P<0.05)$. This appeared to be due to large changes that occurred in a small number of individuals. The susceptibility of LDL to oxidation was measured as the lag phase following in vitro exposure to $\mathrm{Cu}$. There were no consistent between- or within-effects of diet on these two measures.

The haemostatic variables measured at 0,8 and 16 weeks are shown in Table 5. There were no consistent between- or within-effects of diet on fasting factors VIIc or VIIag, fibrinogen or tPA. Female subjects were found to have consistently higher fibrinogen concentrations than male subjects across all three time points (results not shown; $P<0 \cdot 001$ ). Postprandial measurements of factors VIIc and VIIag are shown as their summary measures (AUC and integrated AUC) in Table 5. There were no significant differences between the two diets with regard to their effects on postprandial factor VII activation or levels of antigen, with postprandial factor VIIc levels tending to be lower on both the MUFA diets than at baseline. Within the high-MUFA group, postprandial factor VIIc values were significantly lower at 16 weeks on the diet compared with baseline $(P<0 \cdot 01)$.

Whole blood responses to ADP, collagen and arachidonic acid were measured at baseline, and at 8 and 16 weeks of the MUFA diets (Fig. 1). Repeated-measures ANOVA revealed different effects of the two MUFA diets (time $X$ diet interactions) in the platelet aggregatory response for ADP $(P<0.01)$ and arachidonic acid $(P<0.05)$; this reflected differences in the sustained response to the agonists between the diets at 16 weeks. Decreases in platelet aggregation were observed in response to all three agonists by 8 weeks of both MUFA diets; these differences were significant for ADP and arachidonic acid for both moderate-MUFA $(P<0.05$ and $P<0.01$ respectively) and highMUFA diets $(P<0 \cdot 01$, both agonists). The reduced aggregatory responses to ADP and arachidonic acid at 8 weeks $(P<0.01)$ were maintained by 16 weeks for the highMUFA group only. The aggregation response to both ADP and collagen was consistently and significantly lower in the male than the female subjects $(P<0 \cdot 05$; results not shown), although there were no significant gender differences in response to the MUFA-enriched diet.

\section{Discussion}

The purpose of the present study was to investigate whether the previously reported beneficial effects of substituting dietary SFA with MUFA observed in subject groups at increased risk of CHD ( $n$ 25) are also seen in young normolipidaemic subjects. In addition, the aim

Table 3. Phospholipid fatty acid classes at baseline (end of reference diet) and during monounsaturated fatty acid (MUFA) diets† (Mean values and standard deviations)

\begin{tabular}{|c|c|c|c|c|c|c|c|c|c|c|c|c|}
\hline \multirow[b]{2}{*}{ Time... } & \multicolumn{6}{|c|}{ Moderate-MUFA group ( $n$ 14) } & \multicolumn{6}{|c|}{ High-MUFA group ( $n$ 15) } \\
\hline & \multicolumn{2}{|c|}{ MO } & \multicolumn{2}{|c|}{ M8 } & \multicolumn{2}{|c|}{ M16 } & \multicolumn{2}{|c|}{ Mo } & \multicolumn{2}{|c|}{ M8 } & \multicolumn{2}{|c|}{ M16 } \\
\hline \multicolumn{13}{|c|}{ Phospholipid fatty acid classes (g/100 g fatty acids) } \\
\hline Total SFA & $44 \cdot 5$ & $2 \cdot 1$ & $44 \cdot 1$ & $1 \cdot 3$ & 43.9 & $1 \cdot 2$ & $44 \cdot 7$ & $1 \cdot 7$ & $44 \cdot 1$ & $1 \cdot 6$ & $44 \cdot 1$ & $2 \cdot 0$ \\
\hline Total MUFA & $11 \cdot 6$ & $1 \cdot 1$ & $11 \cdot 7$ & 0.9 & $11 \cdot 2$ & 0.9 & 11.4 & $1 \cdot 1$ & $12 \cdot 3^{*}$ & 1.9 & $12 \cdot 6^{\star \star}$ & 1.6 \\
\hline \multicolumn{13}{|c|}{ Selected individual fatty acids ( $\mathrm{g} / 100 \mathrm{~g}$ fatty acids) } \\
\hline $16: 0$ & 30.9 & $2 \cdot 0$ & $29 \cdot 7$ & 1.5 & $30 \cdot 0$ & $1 \cdot 8$ & 30.00 & 2.0 & $29 \cdot 0^{* *}$ & $2 \cdot 1$ & $29 \cdot 2^{*}$ & $2 \cdot 2$ \\
\hline $18: 1 n-9$ & 11.0 & 1.0 & 11.0 & 0.9 & $10 \cdot 6$ & 0.9 & $10 \cdot 8$ & 1.0 & 11.6 & 1.6 & $11.9^{*}$ & 1.6 \\
\hline $18: 2 n-6$ & $25 \cdot 1$ & 2.9 & 24.5 & 2.5 & $25 \cdot 2$ & $2 \cdot 4$ & $25 \cdot 6$ & $2 \cdot 0$ & $23 \cdot 9^{\star \star}$ & $2 \cdot 3$ & $23 \cdot 6^{\star \star}$ & $2 \cdot 2$ \\
\hline
\end{tabular}

M0, baseline; M8, week 8; M16, week 16; SFA, saturated fatty acid; PUFA, polyunsaturated fatty acid.

Mean values were significantly different from those at baseline (M0): ${ }^{\star} P<0.05,{ }^{\star \star} P<0.01$.

†For details of subjects, diets and procedures, see Table 1 and p. 598. 

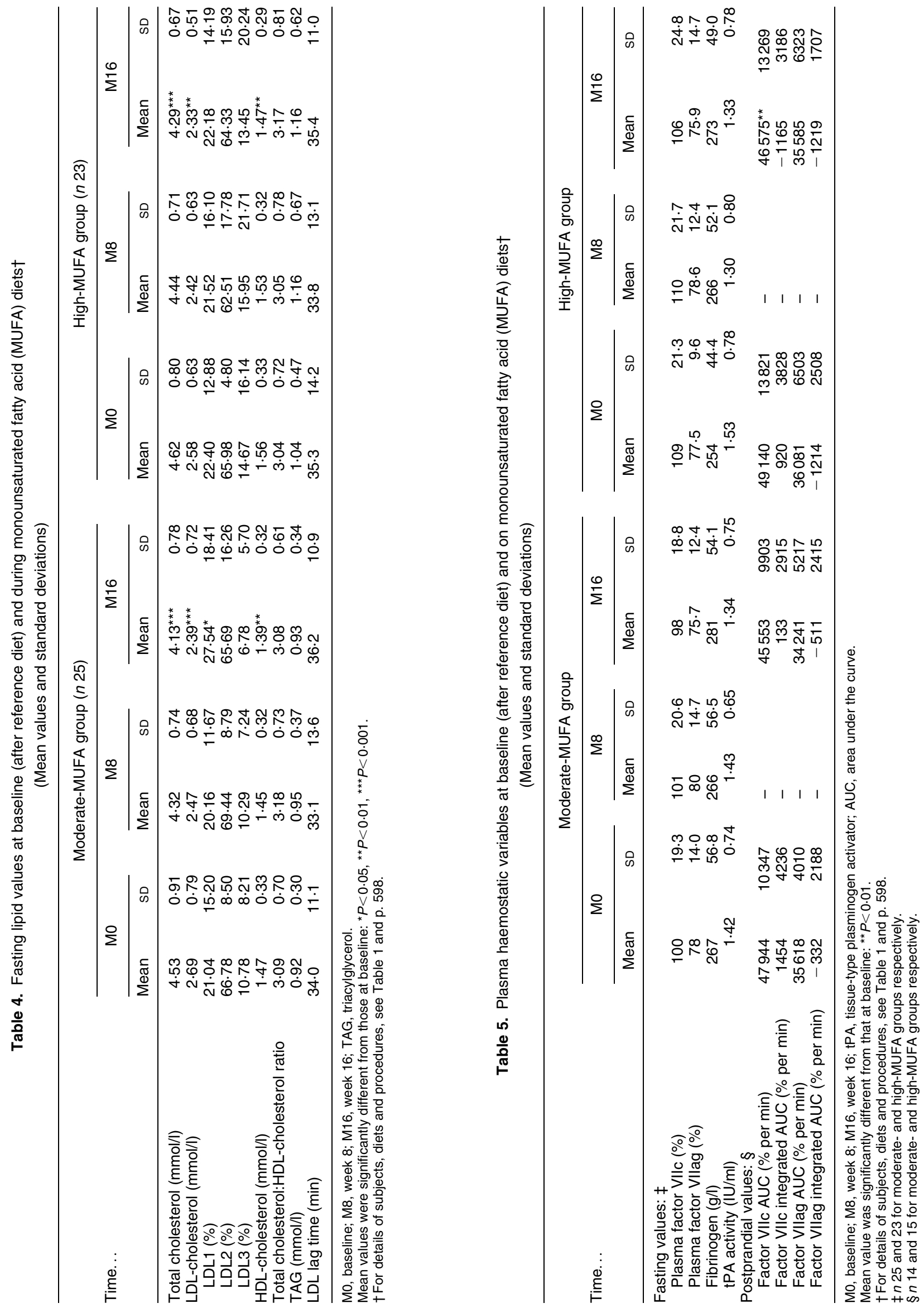

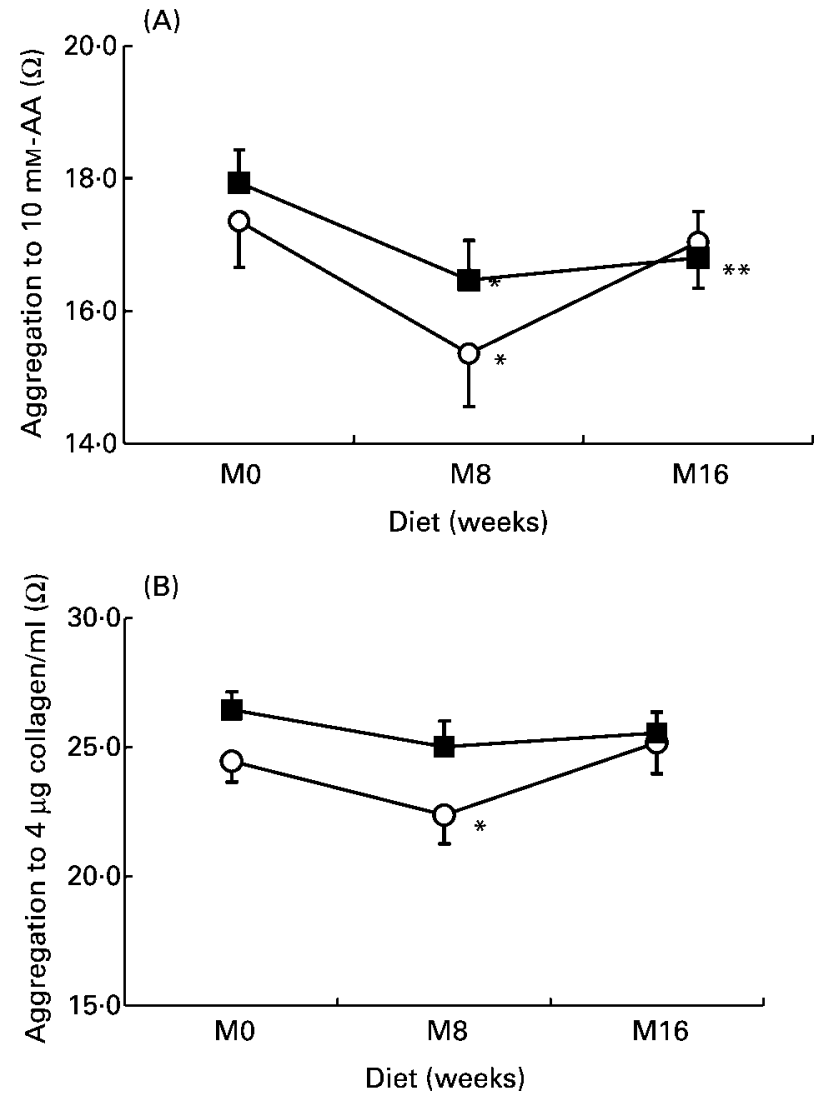

(C)

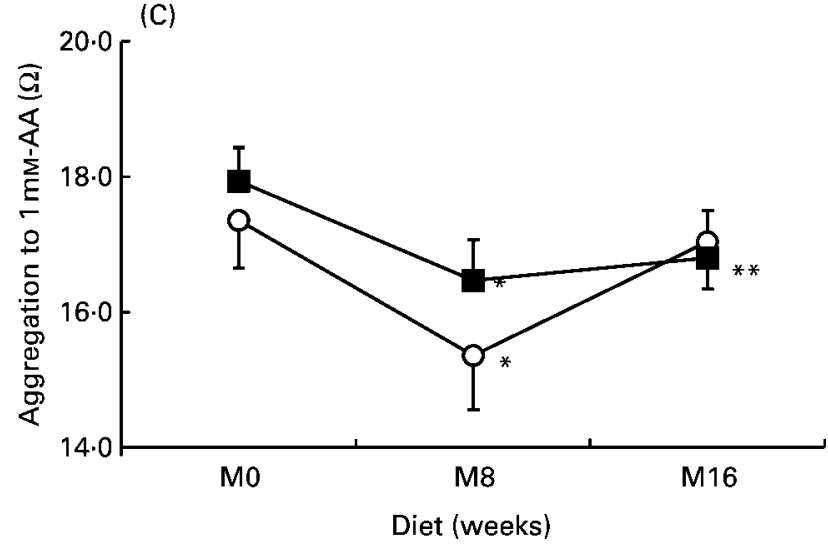

Fig. 1. In vitro platelet aggregatory responses $(\Omega)$ in response to: (A), ADP $(10 \mu \mathrm{M})$; (B), collagen $(4 \mu \mathrm{g} / \mathrm{ml})$; (C), arachidonic acid (AA, $1.0 \mathrm{~mm}$ ). O, Moderate-MUFA diet $(n$ 12); $\boldsymbol{\square}$, high-MUFA diet ( $\mathrm{n} 13)$. M0, baseline (end of reference diet period); M8, week 8; M16, week 16. For details of subjects, diets and procedures, see Tables 1 and 2 and p. 598. Values are means with their standard errors shown by vertical bars. Mean values were significantly different from those at baseline: ${ }^{*} P<0.05,{ }^{* \star} P<0.01$.

was to determine whether beneficial atherogenic and thrombogenic responses are observed at both moderate and high levels of SFA substitution with MUFA and when diets are consumed in the long term.

The dietary interventions were successful in achieving significant increases in cis-MUFA and decreases in SFA when compared with the run-in (reference) diet, the fatty acid composition of which was based on average UK intakes. Significantly lower SFA intakes were achieved on the high-MUFA compared with the moderate-MUFA diet, although target differences in MUFA intakes between the two groups were not quite achieved, at least according to the dietary records that were recorded. Nevertheless, the biomarker results (plasma phospholipid fatty acid compositions) indicated that changes in both SFA and MUFA concentrations within circulating lipids were more marked in the high-MUFA than the moderate-MUFA group.

There were no differences between the two diets with regard to their effects on fasting blood lipids, and significant reductions in total cholesterol and LDL-cholesterol were observed for both MUFA diets. Surprisingly the \% reductions were greater for the moderate- than the highMUFA group. Although the subjects were stratified into the two groups according to their LDL-cholesterol values at screening, it was notable that the moderate-MUFA group ended the run-in diet with a slightly higher LDL concentration than the high-MUFA group. This may indicate greater sensitivity to SFA substitution, which might explain these findings. During both MUFA diets, there were small but significant reductions in HDL-cholesterol. Although there are conflicting results concerning effects on HDLcholesterol of substituting SFA with MUFA (Kris-Etherton et al. 1999; Williams et al. 1999), recent meta-analysis (Mensink \& Katan, 1992) indicates that a slight reduction in HDL would be expected in our present subjects. However, the reductions we observed (approximately $0.10 \mathrm{mmol} / \mathrm{l}$ ) were greater than that predicted from published equations (approximately $0.05 \mathrm{mmol} / \mathrm{l}$ ) (Yu et al. 1995). Female subjects on both diets showed a tendency to show greater HDL reductions on the MUFA diets than did the male subjects and the reductions mostly occurred between week 8 and 16 . It is possible that this change reflects seasonality, but this is made less likely by the fact that other measures that would be expected to show similar seasonal trends (e.g. body weight, plasma TAG) were notably stable in our subjects. We suggest that an explanation, both for the lack of an LDL 'dose-response' to the MUFA diets and the fall in HDL observed on both diets, may be the low pre-intervention LDL-cholesterol levels in this young group. Many of the subjects studied had low LDL-cholesterol levels at baseline (some as low as $1.78 \mathrm{mmol} / \mathrm{l}$ ), so that the capacity to reduce LDL further when a high-MUFA diet is fed may be limited. It might also be expected that at low LDL concentrations, the rate of reverse cholesterol transport and thereby the generation of HDL-cholesterol would be reduced, providing an explanation for greater HDL-cholesterol reduction with time on the diet.

The present study found no evidence for effects of level of dietary MUFA on fasting coagulation (factors VIIc and VIIag, fibrinogen) or fibrinolytic (tPA) markers. This is in agreement with the majority of previously reported work, although recent evidence has suggested that fasting factors VIIc and VIIa may be beneficially reduced following consumption of MUFA-enriched diets compared with a habitual diet rich in SFA (Larsen et al. 1999). Similarly, a recent comparison of urban and rural populations in western Sicily (Avellone et al. 1998) provided evidence of a significant reduction in factor VIIc and plasminogen activator inhibitor-1 activity when the urban population changed to 
a 'Mediterranean type' diet for 8 weeks, and conversely there were significant increases in factor VIIc, t-PA antigen, plasminogen activator inhibitor- 1 activity and fibrinogen in the rural population when they consumed the 'urban type' diet. Subjects showed significant increases in plasma TAG in response to ingestion of the standard test meal containing $45 \mathrm{~g}$ fat (Silva et al. 2003). The postprandial activation of factor VII (factor VIIc) did not differ between the two diets, with postprandial factor VIIc tending to be lower than at baseline for both diets. This attenuating effect was most marked in the high-MUFA group, in whom postprandial factor VIIa levels were significantly reduced compared with baseline. The results are in agreement with our own previous finding (Roche et al. 1998) and those of others (Larsen et al. 1999); this suggests an attenuating effect of chronic MUFA feeding on postprandial activation of factor VII that is the opposite effect to that reported with acute ingestion of MUFA in single test meals (Oakley et al. 1998; Sanders et al. 1999). Although postprandial TAG responses to a standard test meal did not differ on the two diets, we have shown that postprandial apolipoprotein B-48 responses were markedly affected by MUFA ingestion, with responses in the order: reference diet $>$ moderate-MUFA diet $>$ high-MUFA diet (Silva et al. 2003). The results suggest that chylomicron particle number is substantially decreased on the high-MUFA diet and could explain attenuated postprandial factor VII activation via reduced contact activation of factor VII by chylomicrons postprandially.

The high-MUFA diet also resulted in sustained attenuation of platelet aggregation at 16 weeks. In the present study, aggregation in response to all three agonists used was reduced at 8 weeks on both diets. However, these effects were only sustained in the high-MUFA group. Other studies have provided conflicting findings for effects of substitution of SFA with MUFA on platelet aggregation. Sirtori et al. (1986) reported significant decreases in aggregation in response to an 8-week olive oil-intervention diet that provided $17.5 \%$ energy as MUFA. Other studies reported no effect of high-MUFA diets on platelet aggregation induced by collagen (McDonald et al. 1989; Freese et al. 1994) or by ADP (Freese et al. 1994), although a recent study showed increased platelet aggregation in response to collagen when SFA were substituted with either dietary linoleic or oleic acids (Turpeinen et al. 1998). In the present study, particular care was taken to control the nutritional and pharmacological status of the subjects, both chronically in relation to fatty acid intervention itself (Nydahl et al. 2003), and acutely in terms of the standardisation of conditions prior to and during blood collection and measurement of platelet aggregation. The mechanisms by which increased dietary MUFA may influence platelet aggregation are not clear, but we speculate that these may be related to changes in membrane composition and viscosity, eicosanoid metabolism or signal transduction pathways. The possibility that this effect may be due to changes in platelet membrane fatty acid composition is supported by the observation that, like the platelet aggregation responses, changes in circulating lipid compositions were most marked and sustained throughout the 16 weeks in the high-MUFA group. A limitation is that we did not measure platelet membrane phospholipid fatty acid composition directly. However, circulating phospholipids provide the source of membrane phospholipids for platelets and can be regarded as a good surrogate for the platelet. Overall, the results suggest that changes in phospholipids composition that are achieved on moderate-MUFA diets are less marked than on a high-MUFA diet and this reduces the long-term impact of the diet on platelet aggregation.

The present study has shown that previously observed benefits of MUFA-enriched diets on blood lipids in older subjects are also seen in young healthy individuals in the long term, and that benefits are observed with both moderate and high levels of MUFA substitution for SFA. High level substitution of dietary SFA with MUFA also resulted in beneficial effects on platelet aggregation and postprandial factor VII activation, which may be expected to attenuate thrombotic responses in vivo; for the moderateMUFA diet these effects were less marked and sustained than for the high-MUFA diet. Although we observed the expected reductions in total cholesterol and LDL-cholesterol, it was surprising to note that these were not related in a dose-response fashion to the two levels of SFA or MUFA intake. Furthermore, the more marked reductions in HDL-cholesterol than expected indicate that beneficial increases in the total cholesterol:HDL-cholesterol ratio may be more difficult to achieve in young healthy individuals with low baseline LDL-cholesterol values.

\section{Acknowledgements}

The present study was funded by the Food Standards Agency, UK, and M. C. N. also received financial support from The Swedish Foundation for International Co-operation in Research and Higher Education. The authors acknowledge the contribution of Van den Bergh Foods Ltd, UK, in providing the experimental fats, and United Biscuits Ltd, UK, for manufacturing the snack foods. They also wish to thank M. C. Clark and V. Ilic for their technical assistance in the laboratory, Professor K. Frayn for his supervision, and the staff and students of St George's Hall of Residence, University of Reading, UK, for their invaluable help and co-operation with the study.

\section{References}

Avellone G, Di Garbo V, Cordova R, Scaffidi L \& Bompiani GD (1998) Effects of Mediterranean diet on lipid, coagulative and fibrinolytic parameters in two randomly selected population samples in Western Sicily. Nutr Metab Cardiovasc Dis 8, 287-296.

Aviram M \& Eias K (1993) Dietary olive oil reduces low-density lipoprotein uptake by macrophages and decreases the susceptibility of the lipoprotein to undergo lipid peroxidation. Ann Nutr Metab 37, 75-84.

Callow J, Samra JS \& Frayn KN (1998) Effect of infusion of a triacylglycerol emulsion on low-density lipoprotein composition and oxidizability. Atherosclerosis 137, 115-123.

Cardinal DC \& Flower RJ (1980) The electronic aggregometer: A novel device for assessing platelet behaviour in blood. J Pharmacol Methods 3, 135-158.

Department of Health (1994) Nutritional Aspects of 
Cardiovascular Disease. Report on Health and Social Subjects, no. 46. London: H. M. Stationery Office.

Fielding BA, Callow J, Owen RM, Samra JS, Matthews DR \& Frayn KN (1996) Postprandial lipemia: the origin of an early peak studied by specific dietary fatty acid intake during sequential meals. Am J Clin Nutr 63, 36-41.

Freese R, Mutanen M, Valsta LM \& Salminen I (1994) Comparison of the effects of two diets rich in monounsaturated fatty acids differing in their linoleic/ $\alpha$-linolenic acid ratio on platelet aggregation. Thromb Haemost 71, 73-77.

Friedewald WT, Levy RI \& Frederickson DS (1972) Estimation of the concentration of low density lipoprotein cholesterol in plasma, without use of the preparative ultracentrifuge. Clin Chem 18, 499-502.

Griffin BA, Caslake MJ, Yip BY, Tait GW, Packard CJ \& Shepherd J (1990) Rapid isolation of low density lipoprotein subfractions from plasma by density gradient ultracentrifugation. Atherosclerosis 83, 59-67.

Grundy SM (1986) Comparison of monounsaturated fatty acids and carbohydrates for lowering plasma cholesterol. $N$ Engl $J$ Med 314, 745-748.

Katan MB (1997) High-oil compared with low-fat, high-carbohydrate diets in the prevention of ischemic heart disease. Am J Clin Nutr 66, 974S-979S.

Kelly CN (2000) Effects of monounsaturated fatty acids on haemostatic factors and lipoprotein risk factors in healthy subjects. PhD Thesis, University of Reading.

Kris-Etherton PM, Pearson RA, Wan Y, et al. (1999) Highmonounsaturated fatty acid diets lower both plasma cholesterol and triacylglycerol concentrations. Am J Clin Nutr 70, 1009-1015.

Kris-Etherton PM \& Yu S (1997) Individual fatty acid effects on plasma lipids and lipoproteins: human studies. Am J Clin Nutr 65, $1628 \mathrm{~S}-1644 \mathrm{~S}$.

Larsen LF, Jespersen J \& Marckmann P (1999) Are olive oil diets antithrombotic? Diets enriched with olive, rapeseed or sunflower oil affect postprandial factor VII differently. Am $J$ Clin Nutr 70, 976-982.

Lopez-Segura F, Velasco F, Lopez-Mirandia J, et al. (1996) Monounsaturated fatty acid-enriched diet decreases plasma plasminogen activator inhibitor type 1. Arterioscler Thromb 16, $82-88$.

McDonald BE, Gerrard JM, Bruce VM \& Corner EJ (1989) Comparison of the effect of canola oil and sunflower oil on plasma lipids and lipoproteins and on in vivo thromboxane $\mathrm{A}_{2}$ and prostacyclin production in healthy young men. Am J Clin Nutr 50, 1382-1388.

McDowell IFW, McEneny J \& Trimble ER (1995) A rapid method of measurement of susceptibility to oxidation of lowdensity lipoprotein. Ann Clin Biochem 32, 167-174.

Matthews JNS, Altman DG, Campbell MJ \& Royston P (1990) Analysis of serial measurements in medical research. $\mathrm{Br}$ Med $J$ 300, 230-235.

Mensink RP, De Groot M, van den Broeke L, Severignen-Nobels A, Demacker P \& Katan M (1989) Effect of monounsaturated fatty acids vs. complex carbohydrates on serum lipoproteins and apolipoproteins in healthy men and women. Metabolism 38, $172-178$

Mensink RP \& Katan MB (1992) Effect of dietary fatty acids on serum lipids and lipoproteins. A meta-analysis of 27 trials. Arterioscler Thromb 12, 911-919.

Miller GJ, Stirling Y, Esnouf AP, et al. (1994) Factor VII-deficient substrate plasmas depleted of protein $\mathrm{C}$ raise the sensitivity of the factor VII bio-assay to activated factor VII: an international study. Thromb Haemost 71, 38-48.

Ministry of Agriculture, Fisheries and Food (1996) National Food Survey, 1995. London: H. M. Stationery Office.
National Cholesterol Education Program (1991) Report of the expert panel on population strategies for blood cholesterol reduction. Circulation 83, 2154-2232.

Nydahl MC, Smith RD, Kelly CMN, Fielding BA \& Williams CM (2003) Achievement of dietary fatty acid intakes in longterm controlled intervention studies: approach and methodology. Public Health Nutr 6, 31-40.

Oakley FR, Sanders TAB \& Miller GJ (1998) Postprandial effects of an oleic acid-rich oil compared with butter on clotting factor VII and fibrinolysis in healthy men. Am J Clin Nutr 68, 1202-1207.

Petroni A, Blasevich M, Salami M, Papini N, Montedoro GF \& Galli C (1995) Inhibition of platelet aggregation and eicosanoid production by phenolic components of olive oil. Thromb Res 78, $151-160$.

Reaven P, Parthasarathy S, Grasse BJ, et al. (1991) Feasibility of using an oleate-rich diet to reduce the susceptibility of low-density lipoprotein to oxidative modification in humans. Am J Clin Nutr 54, 701-706.

Roche HM, Zampelas A, Knapper JME, et al. (1998) The effect of chronic olive oil dietary intervention on acute postprandial triacylglycerol and factor VII metabolism. Am J Clin Nutr 68, $552-560$

Sanders TAB, de Grassi T, Miller GJ \& Humphries SE (1999) Dietary oleic and palmitic acids and postprandial factor VII in middle-aged men heterozygous and homozygous for factor VII R353Q polymorphism. Am J Clin Nutr 69, $220-225$.

Silva KDRR, Jones AE, Smith RD, et al. (2003) Chylomicron particle size and number, factor VII activation and dietary monounsaturated fatty acids. Atherosclerosis 166, 73-84.

Sirtori CR, Gatti E, Tremoli E, et al. (1992) Olive oil, corn oil and $n$-3 fatty acids differently affect lipids, lipoproteins, platelets and superoxide formation in type II hypercholesterolemia. Am $J$ Clin Nutr 56, 113-122.

Sirtori CR, Tremoli E, Gatti E, et al. (1986) Controlled evaluation of fat intake in the Mediterranean diet: comparative activities of olive oil and corn oil on plasma lipids and platelets in high-risk patients. Am J Clin Nutr 44, 635-642.

Thompson JM (1992) Blood collection and preparation: pre-analytical variation. In ECAT Assay Procedures: A Manual of Laboratory Techniques, pp. 13-20 [J Jespersen, BM Bertina and F Haverkate, editors]. Dordrecht, The Netherlands: Kluwer Academic Publishers.

Turpeinen AM, Pajari AM, Freese R, Sauer R \& Mutanen M (1998) Replacement of dietary saturated by unsaturated fatty acids: effects of platelet protein kinase $\mathrm{C}$ activity, urinary content of 2,3-dinor-TXB2 and in vitro platelet aggregation in healthy man. Thromb Haemost 80, 649-655.

Volpi N \& Tarugi P (1999) The protective effect on $\mathrm{Cu}^{2+}$ - and AAPH-mediated oxidation of human low-density lipoproteins depends on glycosaminoglycan structure. Biochimie 81, 955-963.

Williams CM, Francis-Knapper JA, Webb D, et al. (1999) Cholesterol reduction using manufactured foods high in monounsaturated fatty acids, a randomised cross over study. $\mathrm{Br} J$ Nutr 81, 439-446.

Yaqoob P, Knapper JA, Webb DH, Williams CM, Newsholme EA \& Calder PC (1998) Effect of olive oil on immune function in middle aged men. Am J Clin Nutr 67, 129-135.

Yu S, Derr J, Etherton TD \& Kris-Etherton PM (1995) Plasma cholesterol-predictive equations demonstrate that stearic acid is neutral and monounsaturated fatty acids are hypocholesterolemic. Am J Clin Nutr 61, 1129-1139.

Zampelas A, Roche H, Knapper JME, et al. (1998) Differences in postprandial lipaemic response between Northern and Southern Europeans. Atherosclerosis 139, 83-93. 